\title{
Relación entre la cultura organizacional, la satisfacción laboral y el engagement en el sector confecciones de Piedecuesta, Santander
}

Relationship among organizational culture, job satisfaction and engagement in the garment industry of Piedecuesta, Santander

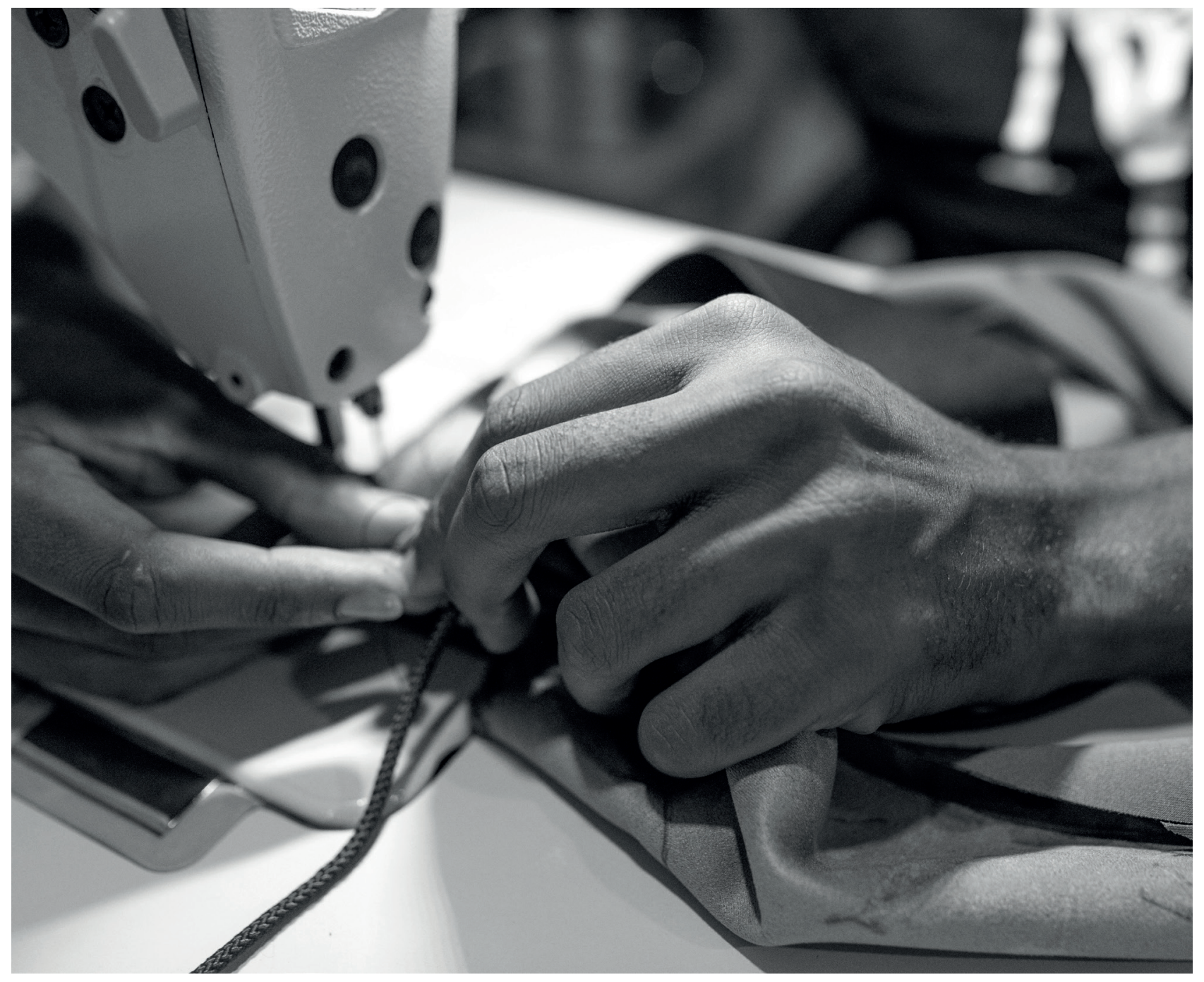

Fuente: Pexels.com Licencia Creative Commons 


\title{
Relación entre la cultura organizacional, la satisfacción laboral y el engagement en el sector confecciones de Piedecuesta, Santander ${ }^{1}$
}

\author{
Relationship among organizational culture, job satisfaction and \\ engagement in the garment industry of Piedecuesta, Santander
}

\author{
Yurani Mendoza-Flórez², Paula Andrea Villamizar-Correa³, Sebastián García-Méndez, Juan \\ Camilo Gutiérrez ${ }^{5}$
}

Artículo recibido en abril 29 de 2020; artículo aceptado en agosto 28 de 2020

\begin{abstract}
Este artículo puede compartirse bajo la licencia Licencia Creative Commons Atribución-NoComercial-Compartir Igual 4.0 Internacional y se referencia usando el siguiente formato: Mendoza-Flórez, Y., Villamizar-Correa, P. A, García-Méndez, S. y Gutiérrez, J. C. (2021). Relación entre la cultura organizacional, la satisfacción laboral y el engagement en el sector confecciones de Piedecuesta, Santander. I+D Revista de Investigaciones, 16(1), 216-224. http://dx.doi.org/10.33304/revinv.v16n1-2021017.
\end{abstract}

\begin{abstract}
Resumen
El documento pretende ahondar en la relación que existe entre la cultura organizacional, la satisfacción laboral y el engagement en el sector de confecciones en Piedecuesta. Para esto se determinaron las dimensiones de cada una de las variables objeto de estudio para la cultura organizacional, las dimensiones de características dominantes, líderes de la organización, estilo gerencial, unión de la organización, énfasis estratégicos y criterio de éxito, que se determinaron utilizando el cuestionario OCAI. Por su parte, el nivel de satisfacción laboral se definió con las condiciones laborales, la participación en las decisiones, la relación con los superiores, el trabajo y el reconocimiento, mediante el cuestionario de satisfacción laboral S20/23. Por su parte, el engagement se determinó con las dimensiones vigor, dedicación y absorción, aplicando el cuestionario UWES-17. Por último, se examinó la relación mediante la correlación de Spearman. Los hallazgos indican que se deben fomentar las buenas relaciones y eliminar las brechas que puedan existir entre trabajador y empleador, para mejorar las relaciones.
\end{abstract}

Palabras clave: Correlación, cultura organizacional, engagement, satisfacción laboral, sector confecciones.

\footnotetext{
${ }^{1}$ Artículo de investigación de tipo correlacional, de corte transversal, con enfoque mixto (cuantitativo y cualitativo), resultado de un proyecto de investigación terminado, perteneciente de ciencias sociales e ingenierías, subárea de gestión industrial, desarrollado en la Universidad de Investigación y Desarrollo (Bucaramanga, Colombia). Dirección: calle 9 n. ${ }^{\circ}$ 23-55, PBX: 6352525. Fecha de inicio: Febrero de 2020. Fecha de Terminación: julio de 2020.

2 Ingeniero Industrial, Universidad de Investigación y Desarrollo (Bucaramanga, Colombia). Juegos y apuestas La Perla S.A Dirección: calle 9 n. ${ }^{\circ} 23-$ 55, PBX: 6352525. ORCID ID: https://orcid.org/0000-0002-2136-7122. Correo electrónico institucional: ymendoza1@udi.edu.co.

3 Ingeniera Industrial, Universidad de Investigación y Desarrollo (Bucaramanga, Colombia). Piedecuestana de Servicios Públicos EPS. Dirección: calle 9 n. $23-55$, PBX: 6352525. ORCID ID: https://orcid.org/0000-0001-6380-3239. Correo electrónico institucional: pvillamizar1@udi.edu.co.

${ }^{4}$ Doctorado en ciencias de la educación, Universidad Cuauhtémoc. Docente investigador del Grupo de Investigación Sinergia, de la Universidad de Investigación y Desarrollo (Bucaramanga, Colombia): Dirección: calle 9 n. ${ }^{\circ}$ 23-55, PBX: 6352525. ORCID: https://orcid.org/0000-0002-6077-0248. Correo electrónico institucional: sgarcia15@udi.edu.co.

${ }^{5}$ Doctorado en Ciencias Administrativas, Instituto Universitario Veracruzano. Docente investigador del Grupo de investigación Sinergia de la Universidad de Investigación y Desarrollo (Bucaramanga, Colombia). Dirección: calle 9 n. ${ }^{\circ}$ 23-55, PBX: 6352525. ORCID ID: https://orcid.org/00000003-0386-1706. Correo electrónico institucional: jgutierrez11@udi.edu.co.
} 
Yurani Mendoza-Flórez, Paula Villamizar-Correa, Sebastián García-Méndez, Juan Camilo Gutiérrez

Relación entre la cultura organizacional, la satisfacción laboral y el engagement en el sector confecciones de Piedecuesta, Santander

\begin{abstract}
This document shows the relationship among organizational culture, job satisfaction and engagement in the garment industry in Piedecuesta. For the development of this investigation, the organizational culture was determined taking into account the following dimensions: dominant characteristics, leaders of the organization, management style, union of the organization, strategic emphasis and criteria of success, by means of the OCAl questionnaire. On the other hand, the level of job satisfaction was defined with the variables working conditions, participation in decisions, relationship with superiors, work, and recognition, by applying the job satisfaction questionnaire S20/23. Likewise, engagement was determined with the dimensions vigor, dedication, and absorption, applying the UWES17 questionnaire. Finally, the relationship was examined using the Spearman correlation. The findings indicate that good relationships should be fostered and the gaps that may exist between worker-employer should be eliminated, in order to improve relationships.
\end{abstract}

Keywords: Correlation, organizational culture, engagement, job satisfaction, garment industry.

\section{Introducción}

El proceso de evolución de la cultura organizacional se ha considerado por más de 40 años como un tema de interés, que pasó de ser solo un elemento en las empresas para convertirse en el componente esencial de estas, especialmente cuando se busca una ventaja competitiva (Barney, 1986; Cantillo-Guerrero y Daza-Escorcia, 2011; Gálvez Albarracín y García Pérez de Lema, 2011; Rodríguez Garay, 2009). Es por esto que desde la dirección empresarial se deben enfocar esfuerzos en estrategias que permitan incentivar al personal e identificar qué tan comprometido está este con las actividades realizadas, a fin de obtener un excelente servicio transmitido al consumidor. Esto otorga una experiencia placentera, por la atención suministrada o bienes adquiridos, lo que logra posicionar el nombre de la organización y genera competitividad en el mercado.

Otro factor fundamental es el recurso humano, dado que este desarrolla las competencias requeridas para construir ventajas competitivas para las organizaciones. Es por esto que la satisfacción laboral es de gran importancia, ya que los trabajadores que presentan un alto nivel de satisfacción tienden a ser más productivos en las empresas, razón clave para realizar un correcto seguimiento (Sánchez et al., 2014) y fomentar la cohesión de equipo (Mendoza-Vega et al., 2020). También se valora como pilar fundamental y de gran importancia para las empresas el engagement, dado que permite que los trabajadores enfoquen de manera positiva las competencias y conocimientos con el fin de alcanzar objetivos organizacionales (Alcántara Quiñones, 2017; Mendoza-Vega et al., 2020).

En este contexto se visualiza que en el ámbito empresarial los constructos descritos son de gran de influencia, teniendo en cuenta las falencias latentes de las organizaciones de los diversos sectores; particularmente, el referente a las confecciones en la competitividad. Esto es validable por el impacto de las importaciones de los productos asiáticos, que causan efectos en las ventas y en los niveles de empleo, lo cual a gran escala afecta a aproximadamente 94.000 personas asociadas directa o indirectamente con la actividad económica (Rodríguez Barajas, 2019). Esto posibilita la realización de la presente investigación en el municipio de Piedecuesta, Santander, cuyo propósito es analizar la relación entre la cultura organizacional, la satisfacción laboral y el engagement, por medio de la utilización de los cuestionarios OCAI, S20/23 y UWES-17, respectivamente, así como la correlación de Spearman.

\section{Cultura organizacional}

La cultura organizacional se refiere a los comportamientos y a las actitudes que se comparten con otros en el trabajo y determinan el grado en que se encuentra la adaptación laboral. Esto, con base en un esquema de referencia en cuanto a patrones de información, para representar un aprendizaje constante, el cual se enriquece con aportes de los individuos. La cultura organizacional se vuelve duradera a través de ellos $y$, a su vez, las personas enriquecen sus entornos (Zapata y Rodríguez, 2008).

Para objeto de esta investigación, se empleó la definición de cultura organizacional del autor Schein (1999), quien la concibe como:

Un patrón de suposiciones básicas compartidas que el grupo aprende como si resolviera sus problemas de adaptación externa e integración interna, que ha funcionado lo suficientemente bien para considerarse válido, $y$, por lo tanto, se les ha enseñado a los nuevos miembros como la manera correcta de percibir, pensar y sentir en relación a esos problemas.

\section{Tipos de cultura organizacional}

Gómez y Sarsosa (2011) citando a Cameron y Quinn (1999) precisan que la cultura organizacional se categoriza en cuatro diferentes tipos, a saber:

- Clan: Orientación interna y control flexible, caracterizados por los valores y objetivos compartidos, 
Yurani Mendoza-Flórez, Paula Villamizar-Correa, Sebastián García-Méndez, Juan Camilo Gutiérrez Relación entre la cultura organizacional, la satisfacción laboral y el engagement en el sector confecciones de Piedecuesta, Santander

la participación, el desarrollo humano, la cohesión y la lealtad.

- Adhocráctica: Orientación externa y control flexible se fomenta el conocimiento y la creatividad entre los diversos empleados de la organización.

- Mercado: Orientación externa y control estable; se reconoce la productividad y la competitividad como ejes orientadores.

- Jerárquica: Orientación interna y control estable; se hace énfasis en la especialización de las tareas, el establecimiento de metas a largo plazo y la eficiencia.

\section{Dimensiones de la cultura organizacional}

De acuerdo con el cuestionario OCAI propuesto por Cameron y Quinn (2011), las dimensiones de la cultura organizacional se dividen en:

- Características dominantes de la organización

- El estilo de liderazgo

- La gestión de los empleados

- La unión de la organización

- Los ejes estratégicos

- Los criterios para el éxito

\section{Método de medición de la cultura organizacional}

Hernández et al. (2014) afirman que "no existe un modelo perfecto de cultura organizacional; no obstante, es necesario que contenga variables cuantitativas y cualitativas en el marco de proporcionar la validez". Del mismo modo, Vargas (2007) afirma que "no se puede medir la cultura con exactitud, debido a que los resultados pueden generar limitaciones y sesgos". Por su parte, Hofstede (1999), citado en Higuita López (2012) indica que existen diversas formas de estudiar la cultura organizacional, teniendo presentes las múltiples formas de interpretación.

\section{Satisfacción laboral}

Muchos autores han definido la satisfacción laboral como la actitud, perspectiva o sentimiento favorable o desfavorable que tienen los trabajadores hacia el trabajo, como resultado de la experiencia que tienen en la organización (Morillo Moronta, 2006). En tal sentido, si la actitud del empleado es favorable hay consecuencias positivas, ya que facilita la satisfacción con la tarea propia y las capacidades, fomenta el rendimiento, la competitividad, la productividad, mejora el clima laboral y potencializa la autonomía y autorrealización del personal, con lo que se beneficia la organización (Huilcapi-Masacon et al., 2017).

Para efecto de esta investigación, se empleó la definición del autor Morillo Moronta (2006, p. 48), que la identifica como el "grado de concordancia entre las expectativas con respecto al trabajo, las recompensas que le ofrece, el estilo gerencial y las relaciones interpersonales", por medio de las perspectivas de favorabilidad o desfavorabilidad de los trabajadores sobre las actividades que se realizan.

\section{Tipos de satisfacción laboral}

Herzberg (1976) desarrolló la teoría de los dos factores. Esta precisa que existen dos tipos de factores que conforman la satisfacción laboral: "los extrínsecos del entorno de trabajo y los intrínsecos que reflejan las experiencias de los individuos con el mismo".

- Satisfacción por factores intrínsecos o motivacionales: Factores generadores de satisfacción; no obstante, su ausencia no ocasiona insatisfacción. Se encuentran vinculados elementos de cumplimiento de metas, el reconocimiento interno y la satisfacción con la tarea.

- Satisfacción por factores extrínsecos o higiénicos: Factores vinculados a las políticas de la organización, la supervisión, las condiciones de trabajo y las relaciones con los compañeros de trabajo. En tal sentido, se deben fomentar idóneos niveles, teniendo en cuenta que son causal de insatisfacción.

\section{Indicadores de la satisfacción laboral}

Autores como Pichler y Wallace (2009) identificaron un conjunto de dimensiones determinantes en la satisfacción laboral, a saber:

- Relaciones con los superiores: Tiene en cuenta el estilo de liderazgo y las capacidades técnicas y administrativas de los directivos a cargo del personal, así como las cualidades a nivel de relacionamiento interpersonal de estos.

- Condiciones laborales: Hace referencia al ambiente físico laboral, como las condiciones ambientales, la limpieza, la ventilación y el espacio disponible para la realización de las actividades.

- Participación en las decisiones: Tiene como objetivo desarrollar la iniciativa e independencia del empleado. En este punto se observa una relación directa entre la satisfacción y el control de un escenario laboral. Es decir que cuanto mayor control perciba el empleado en el ambiente o situación específica del trabajo, mayor será su satisfacción. Esto sucede porque el hecho de ser partícipe en las decisiones de un equipo de trabajo fomenta en la persona una sensación de inclusión y un sentimiento positivo enfocado en la motivación, la integración y el consenso grupal.

- Trabajo: Se refiere al puesto y a la labor del trabajador. 
Yurani Mendoza-Flórez, Paula Villamizar-Correa, Sebastián García-Méndez, Juan Camilo Gutiérrez

Relación entre la cultura organizacional, la satisfacción laboral y el engagement en el sector confecciones de Piedecuesta, Santander

- Prestaciones recibidas: Se refiere a los incentivos que obtiene el trabajador, como recompensas, el sueldo, la promoción y otros, que impulsan al trabajador a realizar las actividades otorgadas por la empresa, con el fin de generar ingresos para satisfacer las necesidades fisiológicas y el estilo de vida del empleado.

\section{Método de medición de la satisfacción laboral}

Existen diferentes métodos de evaluación para identificar la satisfacción laboral que presentan los trabajadores de una organización. Entre estos, se destaca por su uso el cuestionario S20/23. La versión S20/23 muestra unas instrucciones perceptiblemente más breves y sencillas que la versión S4/82, conservando las alternativas de respuesta en una escala Likert, en la que 1 representa Muy insatisfecho, y 7 Muy satisfecho (Meliá y Peiró, 1989).

El cuestionario S20/23 presenta un nivel favorable de fiabilidad y validez, lo que permite la obtención de una medida global de satisfacción y la descripción de los cinco indicadores de satisfacción laboral (Meliá y Peiró, 1989).

\section{El engagement}

El engagement laboral se concibe como un estado mental positivo y satisfactorio frente al trabajo, determinado por el vigor, la dedicación y la absorción del empleado mientras realiza las actividades laborales (Wefald, 2008). En otros términos, es el sentimiento de responsabilidad, compromiso y motivación de los trabajadores frente al desempeño de su trabajo (Salanova y Schaufeli, 2009).

\section{Dimensiones del engagement}

Las dimensiones del engagement, de acuerdo con Salanova y Llorens (2008), citados en Spontón et al. (2012), son:

- Vigor: Se caracteriza por los elevados niveles de energía y esfuerzo en el trabajo, incluso en los momentos en que se presentan dificultades.

- Dedicación: Referido a la implicación del trabajador en el quehacer de su labor y la manifestación de sentimientos como el entusiasmo, la inspiración, el orgullo y el reto por el trabajo.

- Absorción: Corresponde a la percepción sobre el premio o incentivo recibido por el trabajo bien hecho.

El objetivo final de estas tres dimensiones es lograr obtener cambios favorables para la empresa y para los empleados, así como mejorar las expectativas y percepciones que estos tengan de los puestos de trabajo, con el fin de lograr una alta satisfacción que permita el crecimiento profesional.

\section{Instrumentos de medición de engagement}

La Escala Utrecht de Engagement en el Trabajo (UWES, por sus siglas en inglés) fue el instrumento utilizado para medir el nivel de engagement. El UWES indica, a través de preguntas con enfoques positivos, la existencia y el nivel de los factores vigor, dedicación y absorción como indicadores de engagement (Schaufeli et al., 2002).

\section{Correlación de Spearman}

El coeficiente de correlación de Spearman se puede definir como una medida de agrupación entre variables aleatorias de índole cuantitativo, que no tiene en cuenta el nivel de acuerdo o concordancia entre estas (no paramétrica). Se recomienda utilizar este tipo de correlación cuando los datos presentan valores extremos, dado que esos valores afectan negativamente el coeficiente de correlación de Pearson, o cuando se tienen distribuciones no normales (Santander y Ruiz, 2004).

\section{Metodología}

\section{Tipo de estudio}

Esta investigación es de enfoque cuantitativa, y se basó en un diseño no experimental, de corte transaccional o transversal, en vista de que se realiza en un determinado momento y una sola vez. Así mismo, tiene un alcance descriptivo-correlacional, si se tiene en cuenta que primero se describen los tipos de cultura organizacional, la satisfacción laboral y el engagement, para posteriormente realizar la correlación y así poder determinar cuál es la relación entre las variables de estudio en las empresas del sector confecciones en el municipio de Piedecuesta, Santander.

\section{Participantes}

En el estudio participaron 72 empresas de confecciones en Piedecuesta, Santander. Se utilizó un muestreo probabilístico en poblaciones finitas con un $95 \%$ en nivel de confianza y un $10 \%$ de margen de error, aplicado a la población total de 274 empresas suministradas por Compite360, con una población encuestada de 90 trabajadores. El 87,78 \% de los trabajadores que hicieron parte de esta investigación son mujeres. En cuanto al estado civil, el 31,11 \% de los encuestados son casados; el $50 \%$, solteros; el $15,56 \%$, unión libre; el 1,11 \%, divorciados $y$, finalmente, el 2,22 \%, viudos. Además, el principal nivel de escolaridad es bachillerato $(52,22 \%)$, mientras que en menor medida los empleados tienen una educación primaria $(11,11 \%)$, son tecnólogos $(11,11 \%)$, técnicos $(11,11 \%)$ y profesionales $(14,45 \%)$.

En tal sentido, se resalta que las empresas solo contaban con un solo establecimiento, la cantidad de empleados 
Yurani Mendoza-Flórez, Paula Villamizar-Correa, Sebastián García-Méndez, Juan Camilo Gutiérrez Relación entre la cultura organizacional, la satisfacción laboral y el engagement en el sector confecciones de Piedecuesta, Santander

oscilaba entre 1 y 7 , y son sociedades por acciones simplificadas o limitadas.

\section{Materiales e instrumentos}

Los instrumentos seleccionados para la recolección de información sobre las variables cultura organizacional, satisfacción laboral y engagement fueron los cuestionarios OCAI de Cameron y Quinn (1999), S20/23 de Meliá y Peiró, (1989) y el UWES-17 de Schaufeli et al. (2002), respectivamente, los cuales han sido ampliamente utilizados en diferentes investigaciones en los ámbitos nacional e internacional. Así mismo, se menciona el uso del software SPSS versión 25 para el desarrollo de los diversos procedimientos estadísticos.

\section{Procedimiento}

Para el presente estudio se realizó la medición de la cultura organizacional en 72 empresas del sector confecciones en Piedecuesta, Santander, a partir del cuestionario OCAl. Posteriormente, se evaluó la satisfacción laboral mediante el instrumento S20/23, y después el engagement por el cuestionario UWES-17. Finalmente, para determinar la relación existente entre cultura organizacional, satisfacción laboral y engagement, se analizaron los coeficientes de correlación de Spearman entre las variables analizadas.

\section{Resultados}

Los resultados presentados a continuación relacionan la cultura organizacional, la satisfacción laboral, el engagement $y$, finalmente, los coeficientes de correlación de Spearman para las variables objeto de estudio.

\section{Cultura organizacional}

Los resultados indican que 72 empresas cuentan con un alto nivel de cultura organizacional, debido a que cumplen con los requisitos del personal que labora en la organización (ver Tabla 1). Al analizar cada tipo de cultura se observa que la variabilidad sobre esta es mínima (ver Tabla 2).

Tabla 1

Cultura organizacional

\begin{tabular}{cc}
\hline Tipos de cultura & Puntaje \\
\hline Clan & 4,49 \\
Adhocrática & 4,17 \\
Mercado & 4,30 \\
Jerarquizada & 4,39 \\
\hline
\end{tabular}

Fuente: Autores
Tabla 2

Cultura Organizacional por unidad de análisis

\begin{tabular}{ccc}
\hline Unidad de análisis & Puntaje & Tipo de cultura \\
\hline Género masculino & 4,59 & Clan \\
Género femenino & 4,48 & Clan \\
Estado civil casado(a) & 4,50 & Clan \\
Estado civil soltero(a) & 4,41 & Clan \\
Estado civil unión libre & 4,51 & Clan \\
Estado civil divorciado(a) & 5,00 & Adhocrática \\
Estado civil viudo(a) & 4,25 & Jerarquizada Mercadeo \\
Universitario & 4,10 & Clan \\
Tecnólogo & 4,55 & Clan \\
Técnico & 4,65 & Clan \\
Bachiller & 4,07 & Clan \\
Primaria & 4,42 & Jerarquizada \\
\hline
\end{tabular}

Fuente: Autores.

De lo anterior se puede concluir que la cultura clan es la cultura dominante en el sector de confecciones en Piedecuesta, Santander, en vista de que obtuvo el mayor puntaje, y este se ve reflejado en los géneros femenino y masculino. A su vez, se refleja en el estado civil casado, soltero, unión libre, y también en el nivel educativo universitario, tecnológico, técnico y bachiller, considerando que la organización es un lugar con un entorno familiar donde las personas comparten entre sus creencias y valores.

\section{Satisfacción laboral}

De manera análoga, la satisfacción laboral de las 72 empresas encuestadas se encuentra satisfecha, debido a que se cuenta con una participación en las decisiones de los procesos que se desarrollan día a día y cumplen con los requisitos del personal que labora en la organización (ver Tabla 3). Al analizar cada dimensión se observa que la variabilidad sobre esta es mínima, puesto que las empresas objeto de estudio cumplen con los requerimientos del empleado con un nivel de formación universitaria, quienes se encuentran en su totalidad muy satisfechos con el trabajo, con las condiciones laborales y con el reconocimiento otorgado por la empresa (ver Tabla 4).

Tabla 3

Satisfacción laboral

\begin{tabular}{lcc}
\hline \multicolumn{1}{c}{ Dimensiones } & Puntaje & Nivel de satisfacción \\
\hline Condiciones laborales & 6,01 & Muy satisfecho \\
$\begin{array}{l}\text { Participación en las decisio- } \\
\text { nes }\end{array}$ & 6,11 & Muy satisfecho \\
Relación con los superiores & 6,07 & Muy satisfecho \\
Trabajo & 6,09 & Muy satisfecho \\
Prestaciones recibidas & 5,79 & Bastante satisfecho \\
\hline
\end{tabular}

Fuente: Autores. 
Yurani Mendoza-Flórez, Paula Villamizar-Correa, Sebastián García-Méndez, Juan Camilo Gutiérrez

Relación entre la cultura organizacional, la satisfacción laboral y el engagement en el sector confecciones de Piedecuesta, Santander

Tabla 4

Satisfacción laboral por unidad de análisis

\begin{tabular}{ccc}
\hline Unidad de análisis & Puntaje & Nivel de satisfacción \\
\hline Género masculino & 5,91 & Bastante satisfecho \\
Género femenino & 6,03 & Muy satisfecho \\
Estado civil casado(a) & 6,21 & Muy satisfecho \\
Estado civil soltero(a) & 6,00 & Muy satisfecho \\
Estado civil unión libre & 5,80 & Bastante satisfecho \\
Estado civil divorciado(a) & 5,93 & Bastante satisfecho \\
Estado civil viudo(a) & 5,20 & Bastante satisfecho \\
Universitario & 6,39 & Muy satisfecho \\
Tecnólogo & 6,33 & Muy satisfecho \\
Técnico & 5,99 & Bastante satisfecho \\
Bachiller & 5,92 & Bastante satisfecho \\
Primaria & 5,87 & Bastante satisfecho \\
\hline
\end{tabular}

Fuente: Autores.

\section{Engagement}

Por otra parte, el nivel de engagement en las empresas de confección es constante, pues, según los resultados de este estudio, se encuentran "casi siempre" y "bastantes veces" comprometidos con el trabajo que realizan, con el propósito de cumplir los objetivos trazados por la compañía (ver Tabla 5).

Tabla 5

Niveles de engagement

\begin{tabular}{ccc}
\hline Dimensiones & Puntaje & Nivel de engagement \\
\hline Vigor & 5,38 & Casi siempre \\
Dedicación & 5,48 & Casi siempre \\
Absorción & 4,75 & Bastantes veces \\
\hline
\end{tabular}

Fuente: Autores.

En cuanto a los resultados que se muestran en la Tabla 6 , se puede inferir que el nivel de engagement es medianamente proporcional al nivel o a la formación académica de los trabajadores, pues el puntaje del universitario es ligeramente mayor al del tecnólogo o técnico, lo cual reafirma lo encontrado en la teoría concerniente a la naturaleza del trabajo. En esta se resalta que, según el cargo o jerarquía, los trabajadores pueden desarrollar mayor satisfacción laboral (Bòria-Reverter et al., 2012), lo cual depende en su mayoría directamente del nivel de formación académico.

Tabla 6

Niveles de engagement por unidad de análisis

\begin{tabular}{ccc}
\hline Unidad de análisis & Puntaje & Nivel de engagement \\
\hline Género masculino & 5,26 & Casi siempre \\
Género femenino & 5,20 & Casi siempre \\
Estado civil casado(a) & 5,36 & Casi siempre \\
Estado civil soltero(a) & 5,17 & Casi siempre \\
Estado civil unión libre & 5,11 & Casi siempre \\
Estado civil divorciado(a) & 5,38 & Casi siempre \\
Estado civil viudo(a) & 4,28 & Bastantes veces \\
Universitario & 5,25 & Casi siempre \\
Tecnólogo & 5,21 & Casi siempre \\
Técnico & 4,86 & Bastantes veces \\
Bachiller & 5,26 & Casi siempre \\
Primaria & 5,17 & Casi siempre \\
\hline
\end{tabular}

Fuente: Autores.

\section{Correlación de Spearman}

Con el fin de establecer la relación entre las variables de estudio (cultura organizacional, satisfacción laboral y engagement), se hallan los coeficientes de correlación de Spearman (ver Tabla 7). Estos coeficientes de correlación se encuentran entre el rango 0,160 y 0,534. Al llevarse a cabo un análisis de los resultados obtenidos, se evidencia que existe un alto grado de relación entre las dimensiones satisfacción laboral y cultura jerarquizada. Dichas relaciones se presentan con la misma dimensión de la variable engagement (dedicación), por lo cual se concluye que las buenas relaciones con los superiores y la participación en las decisiones influyen en la manera que dedican los esfuerzos y empeños laborales en el trabajo, ya que estos permiten que el trabajador muestre iniciativa y motivación por el trabajo bien realizado.

Tabla 7

Correlación entre las variables objeto de estudio

\begin{tabular}{ccccccccc}
\hline Cultura Org. & \multicolumn{4}{c}{ Satisfacción laboral } & \multicolumn{3}{c}{ Engagement } \\
\hline Clan &, $416^{* *}$ &, $354^{* *}$ &, $279^{*}$ &, $370^{* *}$ &, $449^{* *}$ &, $259^{*}$ &, $363^{* *}$ & 0,228 \\
Mercado &, $297^{*}$ &, $301^{*}$ & 0,157 & 0,199 &, $256^{*}$ & 0,219 & 0,199 & 0,160 \\
Adhocrática &, $479^{* *}$ &, $349^{* *}$ &, $254^{*}$ &, $331^{* *}$ &, $509^{* *}$ & 0,230 &, $413^{* *}$ &, $286^{*}$ \\
Jerarquizada &, $518^{* *}$ &, $534^{* *}$ &, $258^{*}$ &, $400^{* *}$ &, $442^{* *}$ & 0,208 &, $345^{* *}$ &, $312^{* *}$ \\
\hline
\end{tabular}

Fuente: Autores.

La tabla anterior permite visualizar que todos los tipos de cultura organizacional presentan una relación positiva con todas las dimensiones de satisfacción laboral y engagement, teniendo presente lo propuesto por Mondragón Barrera, (2014); a saber, la correlación positiva media se encuentra entre 0,11 y 0,50 , y la positiva considerable, entre 0,51 y 0,75 . 
Yurani Mendoza-Flórez, Paula Villamizar-Correa, Sebastián García-Méndez, Juan Camilo Gutiérrez Relación entre la cultura organizacional, la satisfacción laboral y el engagement en el sector confecciones de Piedecuesta, Santander

\section{Conclusiones}

La cultura organizacional en las empresas de confecciones en el municipio de Piedecuesta, Santander, se caracteriza con la cultura tipo clan. Esto se valida por las características dominantes, líderes de la organización, estilo gerencial, unión organizacional y criterio de éxito. En sentido contrario, el énfasis estratégico se identifica con la cultura jerarquizada, por lo cual las organizaciones hacen énfasis en el desarrollo humano por medio de la participación de cada integrante que conforma la entidad, y esto lleva a que se considere como una familia, teniendo claro su correcto funcionamiento.

Además, este sector en Santander considera al personal como un elemento fundamental que forma parte de la organización, razón por la cual los trabajadores se sienten muy satisfechos con la realización de las labores, debido a que la entidad cumple con las expectativas de los colaboradores, permitiendo el buen desempeño de las actividades diarias para el cumplimiento de los objetivos de la empresa en los planos temporales especificados.

Los empleados objeto de estudio presentan un alto nivel de engagement en el momento de ejecutar las diversas funciones y responsabilidades asignadas en las diferentes áreas funcionales, lo cual posibilita inferir que las entregas de producto en proceso y producto terminado se realizan conforme a los tiempos establecidos. De allí se vislumbran elementos de motivación, entusiasmo y felicidad en el ejercicio de su labor.

La relación entre las variables objeto de estudio permite visualizar que los tipos de cultura organizacional presentan una mayor relación con las dimensiones de satisfacción laboral, si se considera que sus puntajes son superiores que los adquiridos por parte del engagement; por consiguiente, se visiona a largo plazo la identificación de variables con potencial relación frente al constructo mencionado, considerando la importancia en el plano organizacional.

\section{Recomendaciones}

Implementar un plan de mejora enfocado en los tipos de cultura organizacional, teniendo en cuenta la generación de escenarios idóneos de operación, con el fin de impactar favorablemente los niveles de satisfacción laboral y engagement, ya que son ejes fundamentales del trabajo en todas las empresas.

Las empresas de confecciones podrían buscar nuevas alternativas de reconocimiento que lleven a la satisfacción de los trabajadores en las labores que realizan, con el fin de impactar favorablemente los niveles de engagement y de cultura organizacional.

Igualmente, sería interesante realizar más estudios en el sector de confecciones en otros municipios de Santander, con el propósito de efectuar procesos comparativos mediados por las estadísticas para ampliar la información y analizar el comportamiento de las variables cultura organizacional, satisfacción laboral y engagement en el sector a nivel departamental.

\section{Referencias}

Alcántara Quiñones, M. L. (2017). Relación entre engagement y satisfacción laboral en el área administrativa de la empresa UNIMAQ S.A Trujillo [trabajo de grado, Universidad César Vallejo]. Repositorio UCV: http://repositorio.ucv.edu.pe/ bitstream/handle/20.500.12692/9883/alcantara qm.pdf?sequence $=4 \&$ isAllowed $=y$

Barney, J. B. (1986). Organizational Culture: Can It Be a Source of Sustained Competitive Advantage? The Academy of Management Review, 11(3), 656. https:// doi.org/10.2307/258317

Bòria-Reverter, S., Crespi-Vallbona, M. y Mascarilla-Miró, O. (2012). Variables determinantes de la satisfacción laboral en España. Cuadernos de Economía, 35(97), 9-16. https://doi.org/10.1016/S0210-0266(12)700183

Cameron, K. S. y Quinn, R. E. (1999). Diagnosing and Changing Organizational Culture: Based on the competing values Framework. John Wiley \& Sons.

Cameron, K. S. y Quinn, R. E. (2011). Diagnosing and Changing Organizational Culture: Based on the competing values Framework (3. ${ }^{\text {a }}$ ed.). John Wiley \& Sons.

Cantillo-Guerrero, E. F. y Daza-Escorcia, J. M. (2011). Influencia de la cultura organizacional en la competitividad de las empresas. Escenarios, 9(1), 18-23.

Gálvez Albarracín, E. J. y García Pérez de Lema, D. (2011). Cultura organizacional y rendimiento de las Mipymes de mediana y alta tecnología: Un estudio empírico en Cali, Colombia. Cuadernos de Administración, 24(42), 125-145.

Gómez, D. y Sarsosa, K. (2011). Características de la cultura organizacional y comunicación interna en una comercializadora de lácteos de Cali. Pensamiento Psicológico, 9(17), 57-68.

Hernández, R., Méndez, S. y Contreras, R. (2014). Construcción de un instrumento para medir el clima organizacional en función del modelo de los valores en competencia. Contaduría y Administración, 59(1), 229-257. 
Yurani Mendoza-Flórez, Paula Villamizar-Correa, Sebastián García-Méndez, Juan Camilo Gutiérrez

Relación entre la cultura organizacional, la satisfacción laboral y el engagement en el sector confecciones de Piedecuesta, Santander

Herzberg, F. (1976). The Managerial Choice: To Be Efficient and to Be Human. Irwin Professional Publishing.

Higuita López, D. (2012). Interiorización de las manifestaciones culturales en los miembros de la organización. Revista Facultad de Ciencias Económicas, 20(2), 127-142. https://doi.org/10.18359/ rfce. 2168

Hofstede, G. (1999). Culturas y organizaciones. El software mental, la cooperación internacional y su importancia para la supervivencia. Alianza Editorial.

Huilcapi-Masacon, M. R., Jácome-Lara, G. A. y CastroLópez, G. A. (2017). Motivación: las teorías y su relación en el ámbito empresarial. Dominio de las Ciencias, 3(2), 311-333.

Meliá, J. L. y Peiró, J. M. (1989). La medida de la satisfacción laboral en los contextos organizacionales. El cuestionario de satisfacción S20/23. Psicologemas, 5, 59-74.

Mendoza-Vega, R. F., Murillo-Murillo, E. G. y GarcíaMéndez, S. (2020). Relación entre satisfacción laboral y engagement en empleados operativos de la empresa de transporte Cotrascal S. A. S. I+D Revista de Investigaciones, 15(1), 69-76. https://doi. org/10.33304/revinv.v15n1-2020008

Mondragón Barrera, M. A. (2014). Uso de la correlación de Spearman en un estudio de intervención en fisioterapia. Movimiento Científico, 8(1), 98-104. https://doi.org/10.33881/2011-7191.mct.08111

Morillo Moronta, I. (2006). Nivel de satisfacción del personal académico del Instituto Pedagógico de Miranda José Manuel Siso Martínez en relación con el estilo de liderazgo del jefe del departamento, las relaciones interpersonales de los miembros adscritos y el sistema de incentivo. Sapiens. Revista Universitaria de Investigación, 7(1), 43-57.

Pichler, F. y Wallace, C. (2009). What are the Reasons for Differences in Job Satisfaction across Europe? Individual, Compositional, and Institutional Explanations. European Sociological Review, 25(5), 535-549. https://doi.org/10.1093/esr/jcn070

Rodríguez Barajas, Y. C. (2019). Textileros de Santander buscan más participación en el mercado. Vanguardia. Com. https://www.vanguardia.com/economia/ local/textileros-locales-buscan-recuperacionparticipacion-en-el-mercado-nacional-NA561852

Rodríguez Garay, R. (2009). La cultura organizacional. Un potencial activo estratégico desde la perspectiva de la administración. Invenio, 12(22), 67-92.
Salanova, M. y Llorens, S. (2008). Estado actual y retos futuros en el estudio del burnout. Papeles del Psicólogo, 29(1), 59-67.

Salanova, M. y Schaufeli, W. (2009). El engagement en el trabajo. Cuando el trabajo se convierte en pasión. Alianza Editorial.

Sánchez, F., Urcia, V. y Oliden, J. (2014). Relación de cultura organizacional y satisfacción laboral. https:// www.academia.edu/33089071/RELACIÓN_DE_ CULTURA_ORGANIZACIONAL_Y_SATISFACCION_ LABORAL_Integrantes

Santander, A. y Ruiz, R. (2004). Relación entre variables cuantitativas. ECIMED.

Schaufeli, W. B., Salanova, M., González-Romá, V. y Bakker, A. B. (2002). The Measurement of Engagement and Burnout: A Two Sample Confirmatory Factor Analytic Approach. Journal of Happiness Studies, 3(1), 71-92.

Schein, E. H. (1999). Sense and Nonsense About Culture and Climate. https://dspace.mit.edu/bitstream/ handle/1721.1/2759/SWP-4091-43770202.pdf

Spontón, C., Medrano, L. A., Maffei, L., Spontón, M. y Castellano, E. (2012). Validación del cuestionario de engagement UWES a la población de trabajadores de Córdoba, Argentina. Liberabit, 18(2), 147-154.

Vargas, J. (2007). La culturocracia organizacional en México.

Wefald, A. J. (2008). An Examination of Job Engagement, Transformational Leadership, and Related Psychological Constructs [tesis de doctorado, Kansas State University]. file:///C:/Users/USUARIO/ Downloads/AndrewWefald2008.pdf

Zapata, A. y Rodríguez, A. (2008). Cultura organizacional. Universidad del Valle. 